\title{
E-igualdad de género y rendimiento académico en entornos virtuales de aprendizaje: un estudio inter-sujetos
}

\author{
Ascensión Palomares-Ruiz ${ }^{1 *}$, Antonio Cebrián ${ }^{1}$ y José A. López-Pina ${ }^{2}$ \\ (1) Universidad de Castilla-La Mancha, Facultad de Educación de Albacete, Departamento de Pedagogía, Pza. de la \\ Universidad 3, Edificio Simón Abril, 02071 Albacete, España, (correo-e: ascension.palomares@uclm.es, \\ antonio.cebrian@uclm.es) \\ (2) Universidad de Murcia, Departamento de Psicología Básica y Metodología, Avda. Teniente Flomesta 5, 30003, \\ Murcia, España, (correo-e: jlpina@um.es) \\ * Autor a quien debe ser dirigida la correspondencia.
}

Recibido Ene. 16, 2020; Aceptado Mar. 18, 2020; Versión final Abr. 5, 2020; Publicado Oct. 2020

\begin{abstract}
Resumen
El objetivo de esta investigación fue verificar si existen diferencias de género estadísticamente significativas en rendimiento académico en varios grupos de alumnos de $2^{\circ}$ curso del grado de maestro de educación primaria en la Facultad de Educación de Albacete de la Universidad de Castilla La Mancha (España). Se evaluó una misma asignatura impartida de dos formas: 1) con una metodología más tradicional basada exclusivamente en las herramientas proporcionadas por el campus virtual y 2) con una metodología que hizo uso de las herramientas del campus virtual junto a otras herramientas TIC, como Padlet y Socrative, de la Web 2.0. Se empleó un método cuasiexperimental con grupo de control no equivalente. Se encontraron diferencias de género estadísticamente significativas. Se concluye que los resultados concuerdan con los datos proporcionados por el Instituto Nacional de Estadística español que constatan una brecha digital de género que está relacionada con la baja matriculación de mujeres en carreras técnicas.
\end{abstract}

Palabras clave: e-igualdad de género; brecha digital de género; e-inclusión; entornos virtuales de aprendizaje (VLE); aprendizaje mediado por TIC

\section{E-gender equality and academic performance in virtual learning environments: an inter-subject study}

\begin{abstract}
The objective of this research study was to examine whether there were significant statistical differences in academic performance between genders in second year primary education master's degree students from the Faculty of Education of Albacete at the University of Castilla La Mancha (Spain). Gender differences were assessed on the same subject taught in two manners: 1) by using a traditional methodology based exclusively on the tools provided by virtual campus tools and 2) by using virtual campus tools along with other Web 2.0 ICT tools such as Padlet and Socrative. This study used a quasi-experimental method with a non-equivalent control group. The results showed that there were statistically significant differences between genders. It is concluded that the results were in agreement with the data provided by the Spanish National Statistics Institute that confirms a digital gender gap that is related to the low enrollment of women in technical careers.
\end{abstract}

Keywords: e-gender equality; digital gender gap; e-inclusion; virtual learning environments (VLE); ICT mediated learning 


\section{INTRODUCCIÓN}

Es posible que se esté produciendo a nuestro alrededor una "revolución" en un mundo en tránsito desde una sociedad de la información hacia una sociedad del conocimiento; sin embargo, para que esta transformación pueda llegar a nuestras aulas y al sistema educativo, ha de superarse la etapa de la "integración" de esas tecnologías emergentes con los sistemas educativos tradicionales, y ha de ser fruto de la aparición de unas pedagogías emergentes, apoyadas en planteamientos no tan nuevos como el constructivismo, el cognitivismo, el aprendizaje significativo, etc., y otros nuevos conceptos formulados tras la aparición de la web y el Internet como el aprendizaje rizomático, el aprendizaje autorregulado, la teoría LaaN, el aprendizaje entre iguales, y el conectivismo que no han terminado de calar en las prácticas educativas tradicionales y que ahora con la incorporación y adecuado uso de las TIC, se pueden estar incubando las condiciones adecuadas para que el caldo de cultivo creado por estas ideas pedagógicas nuevas y antiguas eclosione y acaben de cambiar de una vez por todas, y para siempre, las formas de trabajar en las aulas, desplazando el foco de atención desde la enseñanza al aprendizaje, del profesor al alumno como sujeto activo y no pasivo de su propio aprendizaje (Domingo y Marqués, 2011; Badia et al., 2013; López-Navajas, 2014; Chaves, et al., 2015; García-Valcárcel y Tejedor, 2017; Fernández et al., 2018).

Existen numerosos estudios en torno a diferencias culturalmente establecidas entre géneros y a cómo esas diferencias culturales impulsan menos a las mujeres a las carreras técnicas (Fernández et al., 2018; Sáinz y López-Sáez, 2010; Espejo, 2015), resaltando incluso que, en las dos últimas décadas, ha descendido su presencia (Carlson 2006; Mateos y Gómez, 2019). Algunas de estas investigaciones apuntan a que, frecuentemente, la tecnología y los ordenadores son considerados socialmente como herramientas masculinas (Kekelis el at., 2005; Gil-Juárez et al., 2010; Drabowicz, 2014; González et al., 2017; Gledhill, et al., 2019) y señalan algunos de los factores implicados en dicho rechazo, tales como que las mujeres hacen un menor uso de los videojuegos, siendo estos una puerta de entrada al mundo de la tecnología (Beavis y Charles, 2007; Gil-Juárez et al., 2010).

Estas investigaciones apuntan a la existencia de tres brechas digitales de género, la primera se revela en el acceso a la tecnología y es de carácter cuantitativo, la segunda se manifiesta en el uso que se hace de la tecnología y, la tercera está relacionada con la utilización de los servicios TIC más avanzados que son de carácter cualitativo (Satpathy y Saroj, 2000). La primera brecha digital de género se está reduciendo gracias al mayor uso de la tecnología; de hecho, en los países occidentalizados las mujeres son usuarias de las TIC en cifras cada vez más parecidas, si no superiores, a las de los hombres (Ruiz y Sánchez, 2010). Sin embargo, la segunda y tercera brecha digital de género trascienden al simple acceso y uso de las TIC y al despliegue de habilidades navegadoras básicas o informáticas, y están vinculadas con la infrarrepresentación de las mujeres en sectores estratégicos de la tecnología.

Los datos proporcionados por el Instituto Nacional de Estadística (INE) referidos a la encuesta sobre equipamiento y uso de tecnologías de información y comunicación en los hogares en España constatan que, si bien la primera brecha digital de género se está reduciendo (Tabla 1 y Figura 1). La segunda y tercera brecha digital de género persisten en el tiempo y están relacionadas con la baja matriculación de mujeres en carreras técnicas (Tablas 2 y 3 y Figura 2).

Las Tablas 2 y 3 y la Figura 2 reflejan los datos de la Secretaría General de Coordinación y Seguimiento Universitario del Ministerio de Educación, Cultura y Deporte (2019) respecto a la distribución de los estudiantes de nuevo ingreso al estudio de Grado por ámbito de estudio y sexo donde puede apreciarse que las mujeres se decantan por carreras relacionadas con la educación, la salud y el sector servicios, mientras que los hombres se decantan por las carreras técnicas, ingenierías e informática destacando que en estos estudios que las mujeres apenas llegan al $10 \%$.

Tabla 1: Brecha digital de género, diferencia entre porcentajes de hombres y mujeres en los indicadores de uso de TIC (Datos tomados del INE, 2019).

\begin{tabular}{|l|c|c|c|c|c|}
\hline & 2014 & 2015 & 2016 & 2017 & 2018 \\
\hline Uso de Internet & 3.4 & 3.2 & 3.9 & 1.8 & 1.0 \\
\hline Uso frecuente de Internet & 4.4 & 3.5 & 3.7 & 1.5 & 1.0 \\
\hline Compras por Internet & 5.1 & 3.9 & 6.1 & 4.9 & 2.3 \\
\hline
\end{tabular}




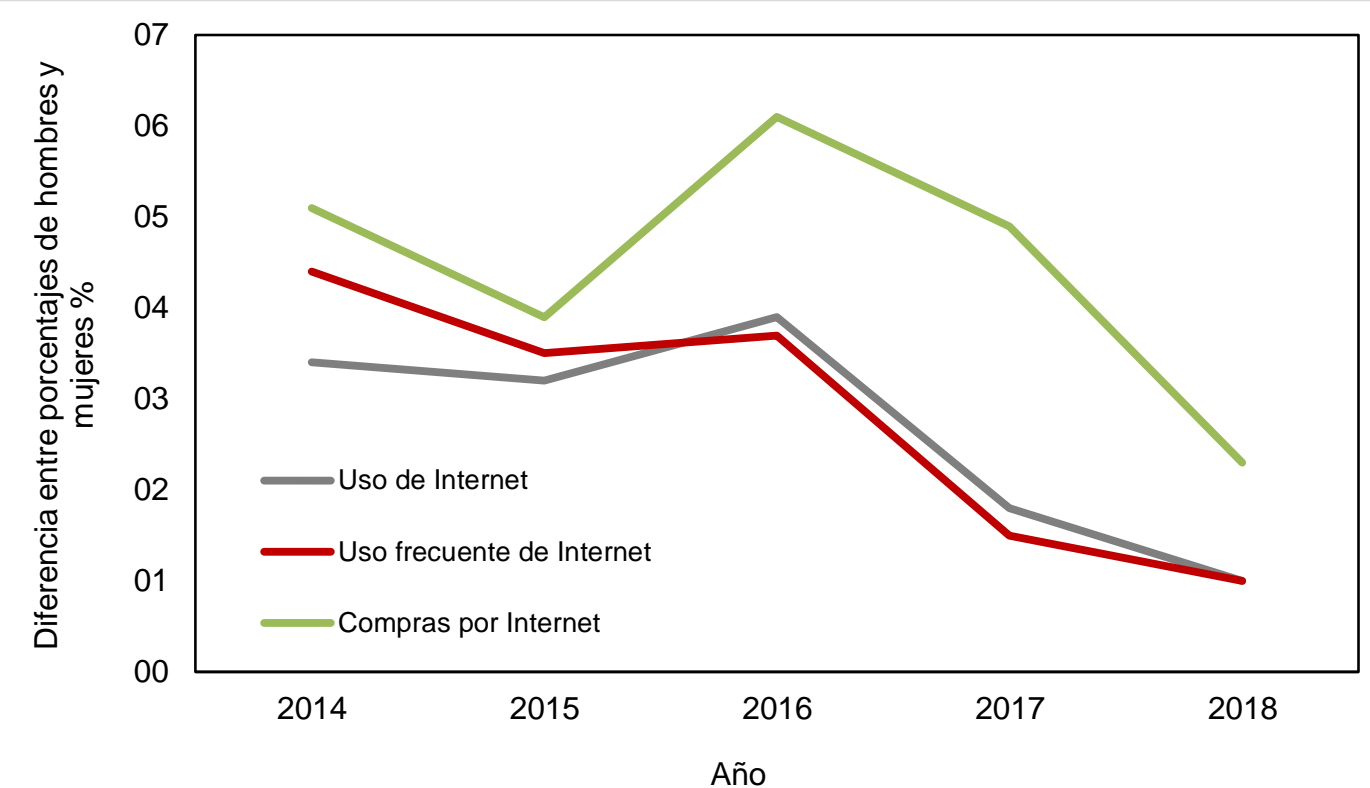

Fig. 1: Brecha digital de género, diferencia entre porcentajes de hombres y mujeres en los indicadores de uso de TIC (Datos tomados del INE, 2019)

Tabla 2: Brecha digital de género (Datos tomados de MECD, 2019)

\begin{tabular}{|l|l|l|l|l|l|l|l|l|}
\hline & Curso 2013/2014 & \multicolumn{2}{l|}{ Curso 2014/2015 } & \multicolumn{2}{l|}{ Curso 2015/2016 } & \multicolumn{2}{l|}{ Curso 2016/2017 } \\
\hline & $\begin{array}{l}\text { Hombres } \\
\%\end{array}$ & $\begin{array}{l}\text { Mujeres } \\
\%\end{array}$ & $\begin{array}{l}\text { Hombres } \\
\%\end{array}$ & $\begin{array}{l}\text { Mujeres } \\
\%\end{array}$ & $\begin{array}{l}\text { Hombres } \\
\%\end{array}$ & $\begin{array}{l}\text { Mujeres } \\
\%\end{array}$ & $\begin{array}{l}\text { Hombres } \\
\%\end{array}$ & $\begin{array}{l}\text { Mujeres } \\
\%\end{array}$ \\
\hline Total Educación & 22.6 & 77.4 & 22.5 & 77.5 & 22.2 & 77.8 & 22.5 & 77.5 \\
\hline Total Salud y servicios sociales & 28.5 & 71.5 & 28.4 & 71.6 & 28.5 & 71.5 & 28.7 & 71.3 \\
\hline $\begin{array}{l}\text { Total Ciencias sociales, periodismo y } \\
\text { documentación }\end{array}$ & 39.1 & 60.9 & 39.6 & 60.4 & 39.3 & 60.7 & 38.7 & 61.3 \\
\hline Total Artes y humanidades & 41.3 & 58.7 & 41.2 & 58.8 & 40.5 & 59.5 & 40.5 & 59.5 \\
\hline Total Negocios, administración y derecho & 48.6 & 51.4 & 48.8 & 51.2 & 48.4 & 51.6 & 48.3 & 51.7 \\
\hline $\begin{array}{l}\text { Total Agricultura, ganadería, silvicultura, pesca, y } \\
\text { veterinaria }\end{array}$ & 56 & 44 & 53.5 & 46.5 & 49.4 & 50.6 & 49.7 & 50.3 \\
\hline Total Ciencias & 52.9 & 47.1 & 54.1 & 45.9 & 52.1 & 47.9 & 52.3 & 47.7 \\
\hline Total Servicios & 52 & 48 & 53.4 & 46.6 & 55.6 & 44.4 & 56.2 & 43.8 \\
\hline Total Ingeniería, industria y construcción & 74.1 & 25.9 & 73 & 27 & 73.9 & 26.1 & 73.8 & 26.2 \\
\hline Total Informática & 87.5 & 12.5 & 88.3 & 11.7 & 88.9 & 11.1 & 87.9 & 12.1 \\
\hline
\end{tabular}

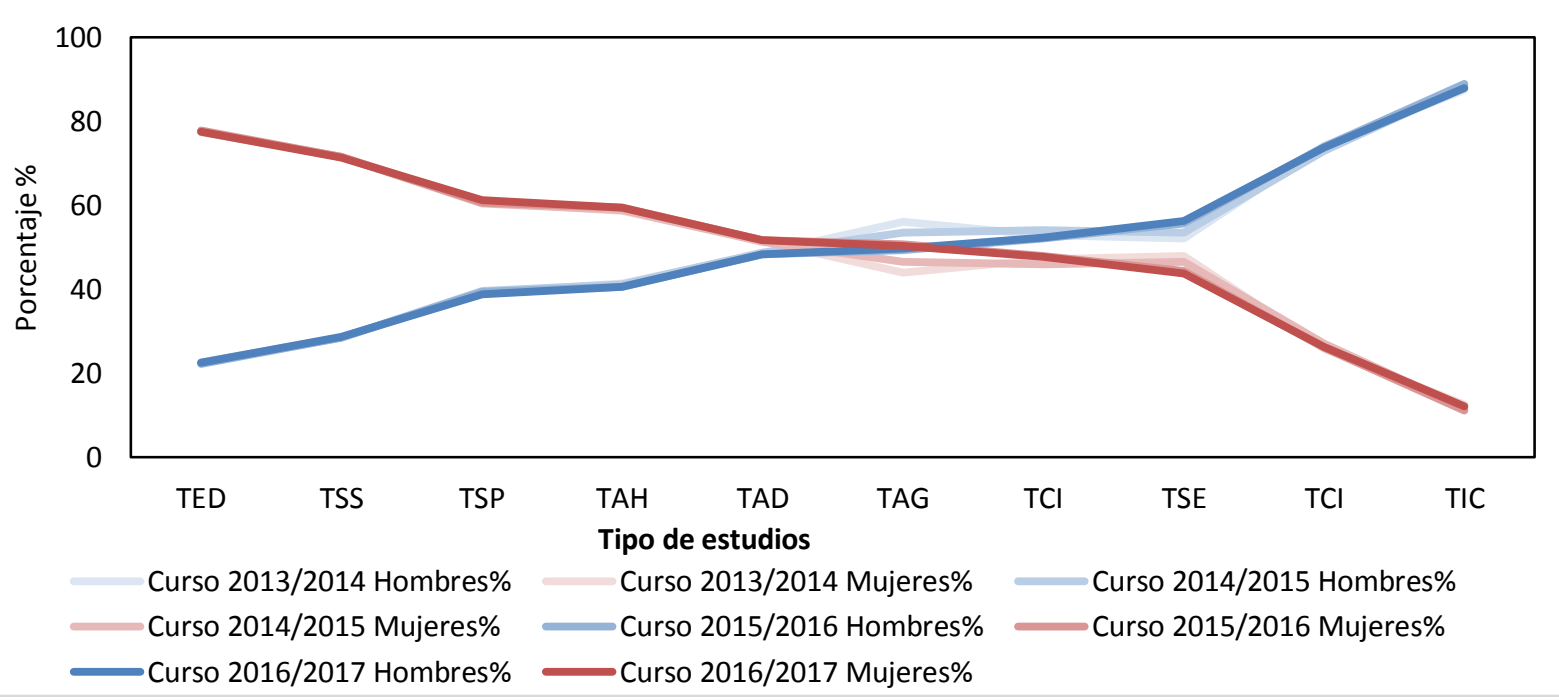

Fig. 2: Distribución de los estudiantes de nuevo ingreso al estudio de Grado por ámbito de estudio y sexo Porcentaje respecto al ámbito de estudio (Datos tomados de MECD, 2019) 
Tabla 3: Ámbitos de estudio y acrónimos utilizados en la Fig:2

\begin{tabular}{|c|l|}
\hline TED & Total Educación \\
\hline TSS & Total Salud y servicios sociales \\
\hline TSP & Total Ciencias sociales, periodismo y documentación \\
\hline TAH & Total Artes y humanidades \\
\hline TAD & Total Negocios, administración y derecho \\
\hline TAG & Total Agricultura, ganadería, silvicultura, pesca, y veterinaria \\
\hline TCI & Total Ciencias \\
\hline TSE & Total Servicios \\
\hline TCI & Total Ingeniería, industria y construcción \\
\hline TIC & Total Informática \\
\hline
\end{tabular}

En una experiencia realizada en la Facultad de Educación de la Universidad de Castilla-La Mancha durante el curso 2016-17, se puso a prueba la hipótesis de posibles diferencias de género comparando el rendimiento académico entre una asignatura impartida en inglés, con una metodología que hacía uso de las herramientas del campus virtual junto a otras herramientas TIC de la Web 2.0 como Padlet y Socrative y la misma asignatura impartida en castellano, en otros grupos por otros profesores diferentes del Departamento de Pedagogía, con una metodología más tradicional basada exclusivamente en las herramientas proporcionadas por el campus virtual de la UCLM, y que constituyeron el grupo de control. El grupo de control estaba constituido por 34 alumnos/as, 23 mujeres y 11 hombres, con edades comprendidas entre los 19 y los 45 años, que pudieron emplear una metodología de trabajo cooperativa/colaborativa que favoreció los trabajos grupales y las exposiciones de los propios alumnos/as. El grupo experimental hizo uso de metodologías de trabajo centradas en el uso de las herramientas TIC de la Web 2.0 como Padlet y Socrative, mostrando un interés y una motivación más elevados, y encontrándose unas calificaciones significativamente más altas en el grupo experimental respecto al grupo de control que utilizó únicamente el campus virtual.

Los entornos virtuales de Aprendizaje (VLE) constituyen un sistema de software proyectado para proporcionar a los profesores la gestión de cursos virtuales. El sistema es capaz de seguir el avance y progreso de los estudiantes, y puede ser gestionado tanto por los docentes como por los propios estudiantes. Aunque inicialmente fueron diseñados para la realización de cursos a distancia, en la actualidad están siendo empleados en cursos presenciales de formación, aunque su concreción tecnológica en los servicios de "campus virtual" reproducen las prácticas y metodologías de una clase tradicional en formato virtual, consiguiendo, por tanto, unos resultados similares (Martínez-Gómez y Marín-García, 2009; Domingo y Marqués, 2011).

En este estudio se plantea si la introducción en el campus virtual de la UCLM de herramientas TIC de la Web 2.0 como Padlet y Socrative favorece el rendimiento académico del alumnado de $2^{\circ}$ curso del Grado de Maestro de Primaria de la Facultad de Educación de Albacete, respecto al uso exclusivo de las herramientas tradicionales del campus virtual de la UCLM, y en verificar si existen diferencias estadísticamente significativas en función del género que permitan suponer la existencia de una brecha digital en los estudiantes. También se establecerán algunas ventajas e inconvenientes del uso de estas nuevas tecnologías en la enseñanza universitaria frente a otros métodos más tradicionales basados en el uso exclusivo del campus virtual.

\section{METODOLOGÍA}

Describimos en este apartado la población y muestra, las variables, el instrumento y el análisis estadístico efectuado en la investigación. Utilizamos una metodología cuasiexperimental con grupo de control no equivalente. Para realizar el análisis estadístico utilizamos 2 programas de análisis de datos de códigos abiertos y gratuitos que existen gracias al trabajo de multitud de desarrolladores de todo el mundo como son PSPP y $R$ con el paquete Rkward.

\section{Población y muestra}

En la investigación se tomó como muestra la población total de alumnos de $2^{\circ}$ curso del Grado de Maestro de Educación Primaria de las asignaturas de Educación y Sociedad durante el curso 2016-2017, con un tamaño de 128 alumnos/as. Los participantes previamente a la investigación ya estaban asignados en 3 grupos distintos 2 del turno de mañana y 1 del turno de tarde, por lo que no se han podido asignar 
aleatoriamente y, por lo tanto, no son grupos equivalentes. Se eligió el grupo 2B para el grupo experimental y el grupo 2C para el grupo de control. El grupo A no participó en la investigación. La muestra quedó constituida por 72 alumnos/as, 34 en el grupo experimental y 38 en el grupo de control. La Tabla 4 presenta los estadísticos descriptivos del rendimiento académico en el grupo experimental (GE) y en el grupo de control (GC) en función del género (hombres $\mathrm{H}$ y mujeres M).

Tabla 4: Estadísticos descriptivos respecto a la variable género

\begin{tabular}{|l|l|l|l|l|}
\hline & \multicolumn{2}{|l|}{ GE } & \multicolumn{2}{l|}{ GC } \\
\hline & $\mathrm{H}$ & $\mathrm{M}$ & $\mathrm{H}$ & $\mathrm{M}$ \\
\hline $\mathrm{M}$ & 8.13 & 8.47 & 6.93 & 7.78 \\
\hline DT & 1.29 & 0.89 & 0.87 & 1.04 \\
\hline Mínimo & 5.2 & 7 & 5.5 & 6.2 \\
\hline Máximo & 9.8 & 9.8 & 9.5 & 9.5 \\
\hline
\end{tabular}

Las medias del grupo experimental tanto de hombres 8.13 como de mujeres 8.47 son más altas que las medias del grupo de control, tanto de los hombres 6.93, como de las mujeres 7.78. Las mujeres del grupo experimental obtienen una media de 8.47 más alta que la de los hombres del grupo experimental 8.13 . Las mujeres del grupo de control obtienen una media de 7.78 más alta que la de los hombres del grupo de control 6.93.

\section{Variables}

Las variables independientes fueron: Metodología de trabajo TIC que adopta dos valores: S (inclusión de herramientas TIC de la web 2.0 Padlet y Socrative en el campus virtual de la UCLM) y N (uso exclusivo de las herramientas del campus virtual de la UCLM), y género (GEN) (H: hombre, y M: mujer). La variable dependiente fue el rendimiento académico de los estudiantes medido a través de las calificaciones de las actas ordinarias de la asignatura Educación y Sociedad (EyS) en el GE y en el GC.

\section{Instrumentos para la recogida de datos}

En el grupo experimental se tuvo en cuenta, en la calificación final, la asistencia a clase 10\%, la elaboración de forma individual de un Portfolio Digital $20 \%$, los trabajos y presentaciones de los/as alumnos/as usando la herramienta Padlet $30 \%$, y una prueba final tipo test con 100 preguntas (50 de verdadero/falso y 50 de opción múltiple) usando la herramienta Socrative. El coeficiente de fiabilidad de las calificaciones obtenidas con la prueba fue $.868_{\overline{1}}$ por lo que se puede asumir la prueba como consistente internamente.

\section{Intervención educativa}

Se pretendió que, la única diferencia entre el grupo experimental y el de control en la intervención educativa, fuese la introducción de herramientas TIC de la Web 2.0 como Padlet y Socrative en el campus virtual de la UCLM en el grupo experimental $2^{\circ} \mathrm{B}$, frente al uso exclusivo de las herramientas tradicionales del campus virtual de la UCLM en el grupo de control $2^{\circ} \mathrm{C}$. El propósito principal fue comparar dos metodologías de trabajo. Una de estas metodologías, más tradicional, estuvo basada exclusivamente en las herramientas proporcionadas por el campus virtual de la UCLM; la otra metodología, haciendo uso también de dicho campus virtual, no se limitó a usar exclusivamente las herramientas proporcionadas por el mismo sino que incluyó otras herramientas TIC de la Web 2.0, tales como videos de Youtube y/o Padlet, que nos permitieron crear posters o murales digitales incorporando textos, fotografías, dibujos, vídeos, audios, hipervínculos, etc. Además, junto a estas herramientas de la Web 2.0 señaladas, se añadió otra: Socrative, utilizada habitualmente para presentar y sintetizar conceptos, realizar una lluvia de ideas, mostrar ejemplos o realizar resúmenes, siendo utilizado como un sistema de respuesta inteligente con los que el profesor puede lanzar preguntas, quizzes y juegos a los que los alumnos pueden responder en tiempo real desde sus dispositivos, ya sea desde un móvil, una tablet o un ordenador portátil.

Tratando de aprovechar todas estas posibilidades, creamos diferentes padlets o muros (Tabla 5) donde los alumnos/as podían bajarse los documentos preparados para cada tema, pudiendo -al mismo tiempo- subir sus presentaciones y documentos de trabajo tanto grupales como individuales. Se trató de utilizar una metodología activa siguiendo los siguientes principios pedagógicos: trabajo colaborativo-cooperativo y autónomo, la reflexión crítica mediante debates e intercambios de ideas para discutir diversas cuestiones, el método de resolución de problemas intentado que los propios estudiantes resuelvan los problemas y expliquen los procesos de resolución a sus compañeros/as usando el lenguaje apropiado y animando a encontrar y discutir distintos procedimientos de solución, siendo el papel del docente el de orientar, guiar, animar y motivar a los alumnos, utilizando en la medida de lo posible materiales y recursos manipulativos, tanto materiales como virtuales, y también se tuvo en cuenta la atención a la diversidad del alumnado con actividades de ampliación y refuerzo. 
Se prepararon unos Padlets introductorios de recursos TIC y otro para los links a los Portfolios Digitales individuales de cada alumno/a, así como 1 padlet para cada uno de los temas en las que se dividió la asignatura, en los mismos se subieron los documentos a trabajar por los participantes, diversos enlaces a webs y videos relacionados con cada tema, los/as alumnos/as también subieron al padlet sus presentaciones grupales y trabajos de cada tema. Cada alumno/a desarrolló de forma individual un Portfolio Digital en el que recogió todas sus producciones e incluyó sus propias reflexiones personales sobre lo aprendido en cada tema, recursos utilizados, etc. Asimismo, cada alumno/a completó la rúbrica de evaluación de su portfolio y la incluyó en su propio Portfolio. Corrección por pares: 1 compañero verificó que los enlaces funcionaban y completó la rúbrica de evaluación de otro Portfolio aparte del suyo propio. El Portfolio final fue un enlace a una red pública (donde no era necesario instalar nada, registrar o recibir una invitación) que contenía los enlaces o rutas a los recursos propios y externos seleccionados por cada alumno/a (Palomares y Cebrián, 2016). Cada alumno/a eligió una plataforma particular donde se sentía más cómodo para albergar su Portfolio (Wiki, Blog, Google Docs, Evernote, etc.) y se incluyeron ejemplos de porfolios con Popplet, Thinglink y Glogster y tutoriales de uso de estas herramientas. Con Socrative, se prepararon para el grupo experimental 2B un test de 100 preguntas, con 50 de verdadero/falso y 50 de opción múltiple. Se usó el Aula de Informática que cuenta con 25 puestos informáticos, aunque algunos/as alumnos/as tuvieron que usar su propio portátil debido a problemas técnicos en algún ordenador.

Tabla 5: Enlaces a los diferentes padlets creados en el grupo experimental EyS

\begin{tabular}{|l|l|}
\hline T0 Introduction and presentation & https://padlet.com/acemar/zunlyto22uax \\
\hline R0 ICT Resources & $\underline{\text { https://padlet.com/acemar/8z2qzag4v5m8 }}$ \\
\hline P0 Digital Portfolio & $\underline{\text { https://padlet.com/acemar/6axwtiocyk3s }}$ \\
\hline T1 Function and tutorial intervention in primary and families & $\underline{\text { https://padlet.com/acemar/n19w27nsmixa }}$ \\
\hline T2 Informational Society and Education & $\underline{\text { https://padlet.com/acemar/qd4bbtbycx53 }}$ \\
\hline T3 ICT in the school context & $\underline{\text { https://padlet.com/acemar/fnnmbw28jzz7 }}$ \\
\hline T4 The influence of context in the classroom & $\underline{\text { https://padlet.com/acemar/sh0s649q8hx4 }}$ \\
\hline T5 Conflict resolution and school coexistence. The mediation & $\underline{\text { https://padlet.com/acemar/1xari105q3uj }}$ \\
\hline T6 Family-school educational strategies. The participation & $\underline{\text { https://padlet.com/acemar/5u54g9bccrpt }}$ \\
\hline T7 Innovation Research Project & $\underline{\text { https://padlet.com/acemar/2iqo8pi0lbw7 }}$ \\
\hline
\end{tabular}

\section{Formulario Evaluación Asignatura}

Para evaluar el grado de satisfacción de los/as alumnos/as respecto a la asignatura y la metodología empleada utilizamos un formulario de Google Docs con 22 ítems que cumplimentaron online de forma anónima 33 de los/as 34 alumnos/as (https://goo.gl/forms/HOY8g60QX3W94cOa2). Los resultados quedan recogidos en las Tablas 6 y 7 , y en la Figura 3.

Tabla 6: Resultados evaluación asignatura EyS

\begin{tabular}{|c|c|c|c|c|}
\hline $\mathrm{N}$ & Questions & SOC & PED & EyS \\
\hline 2 & At what level do you consider this subject is interesting for a future teacher: & 3.52 & 3.52 & 3.52 \\
\hline 3 & $\begin{array}{l}\text { Do you think that the course contents have helped you to better understand the relationship between } \\
\text { Sociology and Pedagogy? }\end{array}$ & 2.76 & 2.76 & 2.76 \\
\hline 4 & Do you consider that the contents have satisfied your expectations? & 2.96 & 2.56 & 2.76 \\
\hline 5 & Do you believe that activities and tasks have been appropriate? & 2.88 & 2.32 & 2.60 \\
\hline 6 & Do you believe that the timeline for activities has been appropriate? & 3.24 & 2.32 & 2.78 \\
\hline 7 & Value the methodology developed for the classes & 3.16 & 2.20 & 2.68 \\
\hline 8 & Would you recommend us to use the same methodology and materials next year? Yes & 2.58 & 1.52 & 2.05 \\
\hline 9 & Value the subject assessment used by the professors. & 3.68 & 3.20 & 3,44 \\
\hline 10 & The assessment and task proposed reflected the most important contents. & 3.20 & 2.88 & 3.04 \\
\hline 11 & What about the documents and readings? & 3.33 & 2,83 & 3.08 \\
\hline 12 & What is your opinion about the coordination between the professors involved in the subject? & 2.84 & 2.84 & 2.84 \\
\hline 13 & Do you consider that there are great differences between the classes of Sociology and Pedagogy? Yes & 3.18 & 3.18 & 3.18 \\
\hline
\end{tabular}


Tabla 6: Continuación.

\begin{tabular}{|c|c|c|c|c|}
\hline 14 & The professor's level of English is similar to other bilingual subjects. & 3.84 & 2.32 & 3.08 \\
\hline 15 & The professor is an expert in the subject he/she teaches. & 3.84 & 3.28 & 3.56 \\
\hline 16 & The professor articulates theory and practice. & 3.77 & 3.17 & 3.47 \\
\hline 17 & The professor seems to be excited about the subject and uses ICTs properly. & 3.79 & 3.54 & 3.67 \\
\hline 18 & The professor's level of English is appropriate to teach this subject in the bilingual degree. & 3.68 & 2.12 & 2.90 \\
\hline 19 & The professor promotes students' participation. & 4.08 & 3.68 & 3.88 \\
\hline 20 & The professor communicates in a clear way, easy to understand. & 3.08 & 2.56 & 2.82 \\
\hline 21 & The professor has solved my doubts and question properly in class or in tutorial time (office or on line). & 3.96 & 3.52 & 3.74 \\
\hline 22 & In general, what is your level of satisfaction with the subject? & 2.84 & 2.84 & 2.84 \\
\hline PRO & TOTAL & 3.34 & 2.82 & 3.08 \\
\hline
\end{tabular}

Tabla 7: Escala de Likert de valoración asignatura EyS

\begin{tabular}{|l|l|l|l|l|}
\hline 5 & 4 & 3 & 2 & 1 \\
\hline Totally interesting & & & & No interest at all \\
\hline Totally appropriate & & & & Totally inadequate \\
\hline Total & & & & Not at all \\
\hline I totally agree & & & & I totally disagree \\
\hline
\end{tabular}

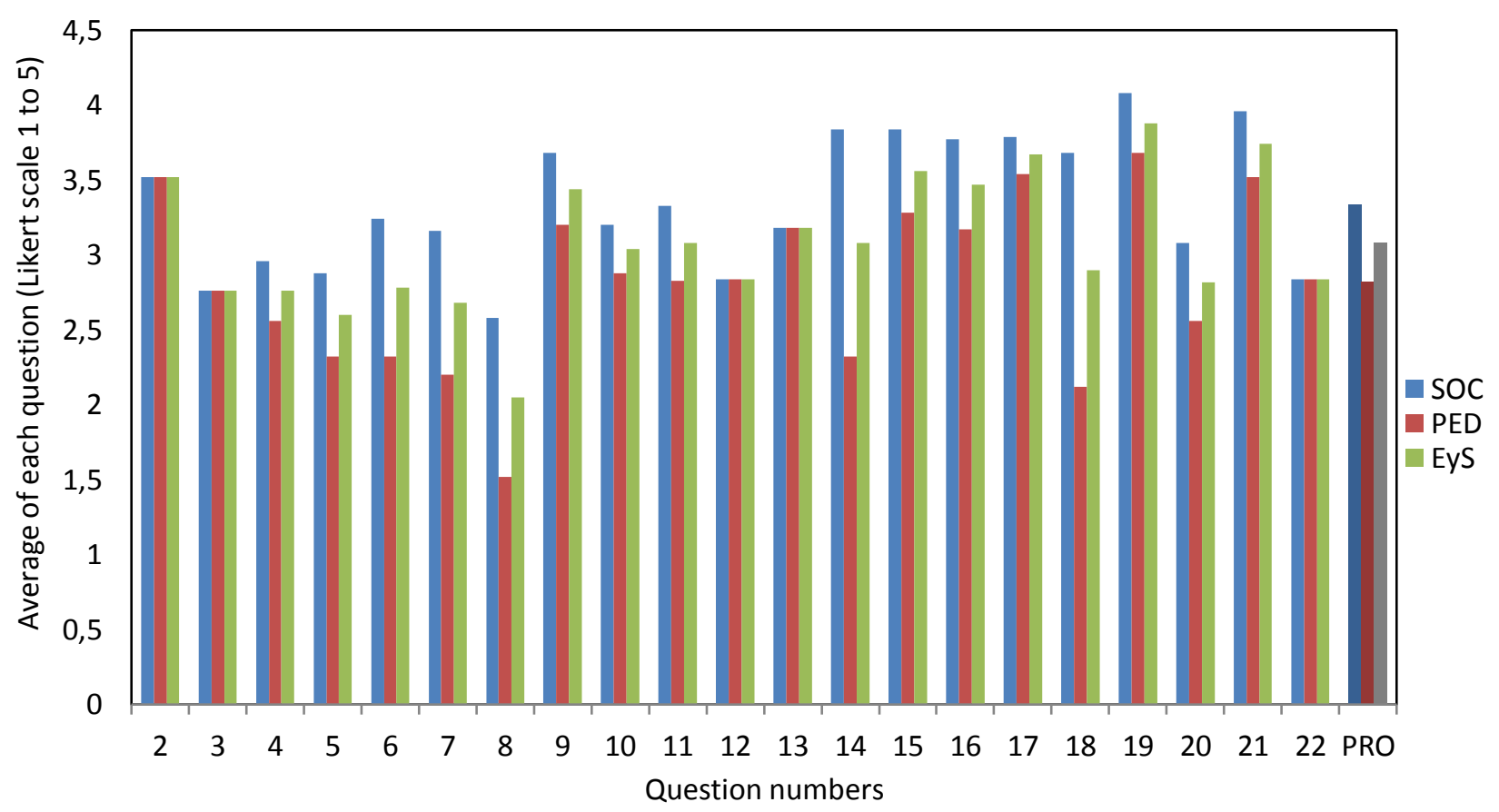

Fig. 3: Resultados evaluación asignatura EyS

\section{RESULTADOS}

La normalidad de la variable dependiente se estudió con la prueba de Kolmogorov-Smirnov. Además, se utilizó un ANOVA en dos sentidos (género x uso de tecnología) para estudiar la significación estadística de la variable dependiente. El nivel de significación se estableció en $\alpha=.05$. En la Tabla 8 podemos observar que la prueba de Kolmogorov-Smirnov no fue significativa ( $K S=1.06, p=.201)$, por lo que se puede asegurar que los resultados en rendimiento académico siguieron una distribución normal. 
Tabla 8: Test de normalidad de Kolmogorov-Smirnov

\begin{tabular}{|l|l|l|}
\hline Prueba Kolmogorov-Smirnov & & Nota_E \\
\hline $\mathrm{N}$ & & 72 \\
\hline Parámetros Normal & Media & 7.82 \\
\hline & Desviación Estándar & 1.17 \\
\hline Diferencias Más Extremas & Absoluto & .12 \\
\hline & Positivo & .12 \\
\hline & Negativo & -.12 \\
\hline Z de Kolmogorov-Smirnov & & 1.06 \\
\hline Sig. Asint. (2-colas) & & .201 \\
\hline
\end{tabular}

Se encontraron diferencias significativas en el rendimiento entre ambos grupos, $F(1,72)=14.193, p<.001$, evidenciando que la utilización de metodologías pueden haber producido un mejor rendimiento en el grupo experimental (Tabla 9). Igualmente, se encontraron diferencias significativas en función del género, $F(1,72)$ $=5.737, \mathrm{p}=.019$, dado que los hombres rindieron peor que las mujeres en ambos grupos, pero no se puede afirmar que ese rendimiento haya sido diferencial en función del método, tal como se aprecia en que la interacción no fue significativa, $F(1,72)=1.060, p=.307$.

Tabla 9: Modelo lineal general univariado de interacción GEN *TIC

\begin{tabular}{|l|l|l|l|l|l|}
\hline \multicolumn{2}{|l|}{ Variable dependiente: No_E } \\
\hline Origen & Tipo III de suma de cuadrados & $g l$ & Media cuadrática & $F$ & Sig. \\
\hline Modelo corregido & $26.787^{\text {a }}$ & 3 & 8.929 & 8.526 & .000 \\
\hline Intersección & 4084.362 & 1 & 4084.362 & 3900.083 & .000 \\
\hline GEN_F & 6.008 & 1 & 6.008 & 5.737 & .019 \\
\hline TIC_F & 14.864 & 1 & 14.864 & 14.193 & .000 \\
\hline GEN_F * TIC_F & 1.110 & 1 & 1.110 & 1.060 & .307 \\
\hline Error & 71.213 & 68 & 1.047 & & \\
\hline Total & 4497.220 & 72 & & & \\
\hline Total corregido & 98.000 & 71 & & & \\
\hline a. $\mathrm{R}$ al cuadrado $=, 273(\mathrm{R}$ al cuadrado ajustada $=, 241)$ & & & \\
\hline
\end{tabular}

\section{DISCUSIÓN}

A la luz de los resultados del ANOVA queda demostrado que las diferencias de medias en el rendimiento académico entre el grupo experimental y el grupo de control son estadísticamente significativas siendo superiores las del grupo experimental, lo que nos permite responder afirmativamente a la pregunta de investigación: ¿La introducción en el campus virtual de la UCLM de herramientas TIC de la Web 2.0 como Padlet y Socrative favorece el rendimiento académico del alumnado de 2o curso del Grado de Maestro de Primaria de la Facultad de Educación de Albacete, respecto al uso exclusivo de las herramientas tradicionales del campus virtual de la UCLM? También se ha comprobado que las diferencias de medias en el rendimiento académico entre mujeres y hombres son estadísticamente significativas. Por otro lado, dado que la interacción entre las variables TIC y GEN no resultó estadísticamente significativa, no se puede afirmar con rotundidad que el empleo de las nuevas tecnologías haya producido un efecto significativo en el rendimiento entre alumnos y alumnas. Sin embargo, sí es cierto que dentro de cada grupo (experimental y control) se produjeron diferencias significativas entre mujeres y hombres; es decir, independientemente de cuál fue la metodología utilizada en ambos grupos, se encontraron diferencias significativas entre mujeres y hombres, siendo superior el rendimiento académico de las mujeres.

Todo ello, enlazando con los datos del INE y, dado que las mujeres son mayoría en las carreras relacionadas con la educación y que, en muchos casos, suelen tener un rendimiento académico superior al de los hombres, como constatamos en la investigación, esto nos permite inferir, no sin cierta cautela, que efectivamente hemos podido entrever una posible brecha digital de género, dado que el empleo de las nuevas tecnologías no ha producido un efecto significativo en el rendimiento académico cuando estudiamos la interacción de la variable GEN con la variable TIC.

En nuestro estudio coincidimos con las investigaciones que apuntan a que las mujeres experimentan grandes dificultades para el manejo de las TIC de forma experta. Pese a que la primera brecha digital de género está desapareciendo, ya que se está equiparando la cifra de usuarios TIC de ambos sexos, sigue estando presente -en la sociedad actual- la segunda brecha digital (Martínez y Serna, 2018), que viene 
determinada por las diferencias en las habilidades que permiten un uso experto de las TIC, lo que tiene una clara influencia en que sólo sean mujeres el 17\% de las alumnas de Ingeniería Informática de España. El problema es consecuencia de diferentes prácticas sociales que ayudan a un menor entusiasmo de las mujeres por las tecnologías, así como del dominio masculino en las áreas donde se crean las nuevas tecnologías (Drabowicz, 2014; López-Navajas, 2014; Mateos y Gómez, 2019).

Recomendaríamos el establecimiento de contextos más favorables para jóvenes y niñas proporcionándoles ejemplos de modelos femeninos en el ámbito de la tecnología, así como el trabajar con el profesorado de institutos y colegios para plasmar acciones de divulgación en actividades informáticas (Ruiz y Sánchez, 2010; Chaves et al., 2015; González et al., 2017). También resulta importante subrayar que, a pesar de que el empleo de los ordenadores como herramienta de relación y comunicación es bastante parejo entre chicos y chicas (Roberts et al., 2005; Quinche y González, 2011), no está sucediendo lo mismo con otros usos del ocio tecnológico como son los videojuegos. Existiendo múltiples estudios que relacionan la brecha digital de género con los videojuegos y con una mengua de oportunidades profesionales y educacionales para las mujeres (Beavis y Charles, 2007; Jenson y De Castell, 2010).

\section{CONCLUSIONES}

De acuerdo al trabajo presentado y a los resultados obtenidos, se pueden plantear las siguientes conclusiones principales: 1) hemos encontrado diferencias de medias estadísticamente significativas entre el grupo de control y el experimental por lo que constamos que la introducción de herramientas TIC de la Web 2.0 en entornos virtuales de aprendizaje (VLE) puede favorecer el rendimiento académico del alumnado respecto metodologías más tradicionales; 2) se han evidenciado diferencias de género estadísticamente significativas independientemente de la metodología utilizada en ambos grupos control y experimental, siendo superior el rendimiento académico de las mujeres en los estudios de Grado de Maestro que entran dentro del ámbito de educación donde predominan ampliamente con más del $77 \%$ de las matriculaciones; 3) se considera necesario motivar a todo el alumnado para que utilicen herramientas digitales externas a la universidad; y 4) al trabajar con grupos de alumnos ya formados, sin poder ser asignados aleatoriamente, la validez externa de la investigación disminuye, con lo que no podemos asegurar que los resultados obtenidos sean generalizables a otros contextos.

\section{REFERENCIAS}

Badia, A., Meneses, J., y Sigalés, C., Teachers' perceptions of factors affecting the educational use of ICT in technologyrich classrooms. doi: http://dx.doi.org/10.14204/ejrep.31.13053, Electronic Journal of Research in Educational Psychology, 11 (3), 787-808 (2013).

Beavis, C., y Charles, C., Would the 'real' girl gamer please stand up? Gender, LAN cafés and the reformulation of the 'girl' gamer. doi: https://doi.org/10.1080/09540250701650615, Gender and Education, 19(6), $691-705$ (2007).

Carlson, S., Wanted: female computer scientists, The Chronicle of Higher Education, 52(19), 35-38 (2006).

Chaves, E., Trujillo, J.M., y López, J., Autorregulación del Aprendizaje en Entornos Personales de Aprendizaje en el Grado de Educación Primaria de la Universidad de Granada, España. doi: 10.4067/S0718-50062015000400008, Formación Universitaria, Vol. 8(4), 63-76 (2015)

Domingo, M., y Marqués, P., Aulas 2.0 y uso de las TIC en la práctica docente. doi: https://doi.org/10.3916/C37-2011-0309, Comunicar: Revista científica iberoamericana de comunicación y educación, (37), 169-175 (2011).

Drabowicz, T., Gender and digital usage inequality among adolescents: A comparative study of 39 countries. doi: 10.1016/j.compedu.2014.01.016, Computers \& Education, 74, 98-111 (2014).

Espejo, R.L. Interacción Simbólica en un Acto de Subjetividad de Género. doi: 10.4067/S0718-50062015000500006, Formación Universitaria Vol. 8(5), 43-58 (2015).

Fernández, F.J., Fernández, M.J., y Rodríguez, J.M., El proceso de integración y uso pedagógico de las TIC en los centros educativos madrileños. doi: 10.5944/educXX1.17907, Educación XX1, 21(2), 395-416 (2018).

García-Valcárcel, A., y Tejedor, F. J., Percepción de los estudiantes sobre el valor de las TIC en sus estrategias de aprendizaje y su relación con el rendimiento. doi: 10.5944/educXX1.19035, Educación XX1, 20(2), 137-159 (2017).

Gledhill, I.M.A., et al. A global approach to the gender gap in mathematical, computing and natural sciences: How to measure it, how to reduce it? doi: https://doi.org/10.17159/sajs.2019/a0305, South African Journal of Science, 115 (34), 1-3 (2019).

Gil-Juárez, A., Feliu, J., y González, A. V., Performatividad Tecnológica de Género: Explorando la Brecha Digital en el mundo del Videojuego. doi.org/10.5565/rev/qpsicologia.758, Quaderns de Psicologia, 12(2), $209-226$ (2010).

González, A.M., Vergés, N., y Martínez, J.S, Las mujeres en el mercado de trabajo de las tecnologías. http://dx.doi.org/10.5477/cis/reis.159.73, Revista Española de Investigación Sociológica, 159, 73-90 (2017).

Instituto Nacional de Estadística (INE), Estadísticas de población, INE, Madrid, España (2019). 
Jenson, J., y De Castell, S., Gender, Simulation, and Gaming: Research review and redirections. doi: https://doi.org/10.1177/1046878109353473, Simulation \& Gaming, 41(1), 51-71 (2010).

Kekelis, L. S., Ancheta, R. W., y Heber, E., Hurdles in the pipeline: Girls and technology careers, Frontiers: A Journal of Women Studies, 26(1), 99-109 (2005).

López-Navajas, A., Análisis de la ausencia de las mujeres en los manuales de la ESO: una genealogía de conocimiento ocultada, doi: 10.4438/1988-592X-RE-2012-363-188, Revista de Educación, 363, 282-308 (2014).

Martínez-Gómez, M., y Marín-García, J.A., Como medir y guiar cambios hacia entornos educativos más motivadores. doi:10.1612/form.univ.4288fu.09, Formación Universitaria, 2(4), 3-14 (209)

Martinez; I., y Serna N., Disparities at the entrance door: gender gaps in elementary school. doi.org/10.1590/s16784634201844184081, Educação e Pesquisa, 44 (2018)

Mateos, S., y Gómez, C., Libro Blanco de las mujeres en el ámbito tecnológico, Secretaría de Estado para el Avance Digital. Ministerio de Economía y Empresa, Madrid, España (2019).

MECD: Ministerio de Educación, Cultura y Deporte (España), Estadísticas de Educación, Secretaría General de Coordinación y Seguimiento Universitario, Madrid, España (2019)

Palomares, A., y Cebrián, A., Una experiencia de Flipped Classroom o Aula Invertida en la Facultad de Educación de Albacete, Tecnología, innovación e investigación en los procesos de enseñanza-aprendizaje, Ediciones Octaedro, pp 2860-2871, Barcelona, España (2016).

Quinche, J.C., y González, F., Entornos virtuales 3D, Alternativa Pedagógica para el Fomento de Aprendizaje Colaborativo y Gestión del Conocimiento en Uniminuto, doi: http://dx.doi.org/10.4067/S0718-50062011000200006,

Formación Universitaria, 4(2), 45-54 (2011).

Roberts, D. F., Foehr, U. G., y Rideout, V. J., Generation M2: Media in the Lives of 8-18-Year Olds, Family Foundation Study, Menlo Park, California, EE.UU (2005).

Ruiz, J., y Sánchez, J., El género como factor influyente en la estrategia para integrar las tic en la práctica docente, Pixel-bit. Revista de medios y educación, 37, 67-76 (2010).

Sáinz, M., y López-Sáez, M., Gender differences in computer attitudes and the choice of technology-related occupations in a sample of secondary students in Spain. doi:10.1016/j.compedu.2009.09.007, Computers \& Education, 54(2), 578587 (2010).

Satpathy, R., y Saroj, K.M., The alarming "gender gap", https://apps.who.int/iris/handle/10665/57783, Bulletin of the World Health Organization: the International Journal of Public Health, 78 (11),1373-1373 (2000) 